\section{How Many Stages in the Coil-to-Globule Transition of Linear Homopolymer Chains in a Dilute Solution?}

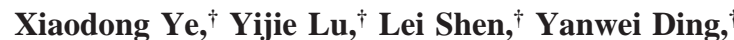 Shilin Liu, ${ }^{\dagger}$ Guangzhao Zhang, ${ }^{\dagger}$ and $\mathrm{Chi} \mathrm{Wu}^{*, \dagger,+}$}

The Hefei National Laboratory for Physical Sciences at Microscale and the Department of Chemical Physics, University of Science and Technology of China, Hefei, Anhui 230026, China, and Department of Chemistry, The Chinese University of Hong Kong, Shatin N.T., Hong Kong

Received January 22, 2007

Revised Manuscript Received May 27, 2007

The coil-to-globule transition of linear polymer chains has attracted much theoretical and experimental attention in the past 50 years, ${ }^{1-11}$ not only due to its fundamental importance in polymer physics but also because of its implication in biology. ${ }^{12-14}$ Thermodynamics of the coil-to-globule transition is fairly understood due to recent experimental advances, such as laser light scattering,,${ }^{7,8}$ fluorescence spectroscopy, ${ }^{9}$ infrared spectroscopy, ${ }^{10}$ and differential scanning microcalorimetry. ${ }^{11}$ In comparison, only a few experimental kinetic studies have so far been reported with controversial results ${ }^{15-18}$ in spite that the transition kinetics has been well studied in theory and computation. ${ }^{18-22}$

Experimentally, Chu et al. ${ }^{15}$ found a slow two-stage process with two transition times on the order of a few minutes. Nakata et al. ${ }^{16}$ showed that the first stage was too fast to be measured in their laser light-scattering spectrometer. Using different chain lengths, Baysal et al. ${ }^{17}$ also measured the first stage, slower than $\sim 30$ s. Recently, Liu et al. ${ }^{18}$ used a stopped-flow device to study the transition kinetics of thermally sensitive linear copolymer chains, poly( $N$-isopropylacrylamide-co-4(1-pyrenyl)butyl acrylate)) (PNIPAM-co-pyrene), in water and found a two-stage kinetics with much shorter characteristic times $\left(\tau_{\text {fast }} \sim 12 \mathrm{~ms}\right.$ and $\tau_{\text {slow }} \sim 270 \mathrm{~ms}$ ). However, the copolymerization of a large amount of hydrophobic fluorescence probes into PNIPAM greatly complicated the problem, and the distribution of pyrene on the PNIPAM chain backbone is problematic. Such a system leads to a less theoretical value.

Theoretically, using the Langevin-equation simulation, Byrne et al. ${ }^{19}$ found three stages: the quick formation of small locally collapsed nuclei on the chain, the growth of these nuclei into clusters at the expense of their surrounding slack segments, and the merging of the clusters until only one single-chain globule is left. Kuznetsov et al. ${ }^{20}$ used the Monte Carlo simulation and the numerical solution on the basis of the Gaussian selfconsistent approach to find four or more different stages. The first three stages are similar to what Byrne et al. found, but the additional fourth stage involves the slow equilibration of a compact collapsed globule. Klushin ${ }^{21}$ showed that the overall characteristic transition time $(\tau)$ could be scaled to the degree of polymerization $(N)$ as $\tau \sim N^{0.93}$ after considering the hydrodynamic interaction. Further, using a phenomenological model, Halperin and Goldbart ${ }^{22}$ also found four similar stages with slightly different meanings, i.e., "pearling", "bridge stretch-

* To whom correspondence should be addressed at the Hong Kong address.

University of Science and Technology of China

$\doteqdot$ The Chinese University of Hong Kong.
Table 1. Laser Light Scattering Characterization of PNIPAM Samples Used at $25.0{ }^{\circ} \mathrm{C}$

\begin{tabular}{ccccc}
\hline sample & $\left\langle M_{\mathrm{w}}\right\rangle(\mathrm{g} / \mathrm{mol})$ & $\left\langle R_{\mathrm{g}}\right\rangle(\mathrm{nm})$ & $\left\langle R_{\mathrm{h}}\right\rangle(\mathrm{nm})$ & $M_{\mathrm{w}} / M_{\mathrm{n}}$ \\
\hline PNIPAM-1 & $1.2 \times 10^{6}$ & 45 & 35 & 1.5 \\
PNIPAM-2 & $2.4 \times 10^{6}$ & 60 & 44 & 1.4 \\
PNIPAM-3 & $3.9 \times 10^{6}$ & 84 & 57 & 1.5 \\
PNIPAM-4 & $7.7 \times 10^{6}$ & 114 & 73 & 1.4 \\
PNIPAM-5 & $2.3 \times 10^{7}$ & 230 & 160 & 1.2
\end{tabular}

ing", "packing", and "merging". Recently, Kikuchi et al. ${ }^{23}$ showed that the hydrodynamic interaction could speed up the transition on the order of milliseconds, and the initial "pearl" formation only took $\sim 5 \%$ of the overall transition time $(\tau)$ even for a long chain. Up to now, there has been no solid experimental data to back such formation of small pearls and the existence of various kinetic stages. A comparison of these theoretical studies leads us to question whether there would be a clear distinction among different stages, especially between the second and third stages even if they did exist.

In this Communication, we report that by using a fast infrared laser heating pulse (width $\sim 10 \mathrm{~ns}$ and $\lambda=1.54 \mu \mathrm{m}$ ) and watersoluble 8-anilino-1-naphthalenesulfonic acid ammonium salt (ANS) free in the solution as a fluorescence probe (due to its sensitivity to its hydrophobic/ hydrophilic surroundings), ${ }^{24}$ we were able, for the first time, to study the fast chain-folding kinetics of linear PNIPAM homopolymer chains in dilute aqueous solutions in terms of both the fluorescence and Rayleigh scattering intensity ( $I_{\mathrm{f} \text {,ANS }}$ and $\left.I_{\text {scattering }}\right)$.

The fast infrared laser heating pulse generated with a $\mathrm{Nd}$ : YAG laser (Spectra Physics, Lab-170, $10 \mathrm{~Hz}$, and pulse width $=10 \mathrm{~ns}$ ) and two Raman cells is adsorbed by the overtone of the $\mathrm{O}-\mathrm{H}$ stretching vibration of $\mathrm{H}_{2} \mathrm{O} .{ }^{25-32}$ A $200-\mathrm{W}$ highpressure mercury lamp (Shanghai Hualun Bulk Factory) was used as the light source for both the fluorescence and Rayleigh scattering experiments. The sample cell consisting of a pair of quartz windows with a $200-\mu \mathrm{m}$ spacer was thermally controlled with a precision of $\pm 0.1{ }^{\circ} \mathrm{C}$ by a heating bath. Each heating pulse raised $\sim 2{ }^{\circ} \mathrm{C}$ in the scattering volume, which is sufficient to change water from a $\Theta$ solvent $\left(\sim 30.5^{\circ} \mathrm{C}\right)$ to a poor solvent and induce the coil-to-globule transition of individual PNIPAM chains. ${ }^{8}$ The detector was placed at a right angle. Each data point was normally averaged over 512 time measurements to improve its signal-to-noise ratio. Using a $50-\mathrm{k} \Omega$ matched resistor, we were able to reach a dead time of less than $\sim 20$ $\mu \mathrm{s}$.

The synthesis of long PNIPAM homopolymer chains was detailed before. ${ }^{8}$ The resultant PNIPAM homopolymer was fractionated by a dissolution/precipitation process in a mixture of dry acetone and dry hexane at the room temperature. The weight-average molar mass $\left(\left\langle M_{\mathrm{w}}\right\rangle\right)$, the gyration of radius $\left(\left\langle R_{\mathrm{g}}\right\rangle\right)$, and the hydrodynamic radius $\left(\left\langle R_{\mathrm{h}}\right\rangle\right)$ of five PNIPAM fractions used are summarized in Table 1 . The polydispersity index $\left(M_{\mathrm{w}} /\right.$ $M_{\mathrm{n}}$ ) was estimated from the relative width $\left(\mu_{2} /\langle\Gamma\rangle^{2}\right)$ of the characteristic line-width distribution $(G(\Gamma))$ measured in dynamic LLS on the basis of $M_{\mathrm{w}} / M_{\mathrm{n}} \sim 1+4 \mu_{2} /\langle\Gamma\rangle^{2} .^{33}$ The final concentration of ANS was determined using an extinction coefficient of $5 \times 10^{3} \mathrm{M}^{-1} \mathrm{~cm}^{-1}$ at $\lambda=350 \mathrm{~nm} .^{34,35}$

Figure 1 shows that the ANS fluorescence intensity $\left[I_{\mathrm{F}}(t)-\right.$ $\left.I_{\mathrm{F}}(0)\right]$ remains a constant after the heating pulse with no PNIPAM in the solution. With PNIPAM, $I_{\mathrm{F}}(t)$ increases rapidly in the first $0.5 \mathrm{~ms}$ and then levels off after $\sim 3 \mathrm{~ms}$. The semilogarithmic plot in the inset clearly shows two distinct 


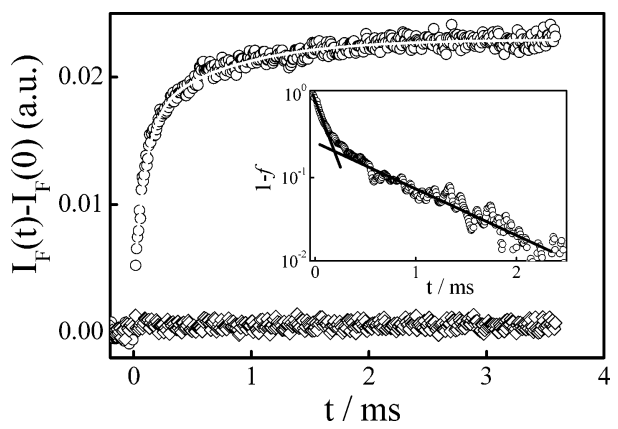

Figure 1. Time dependence of fluorescence intensity changes of ANS $(118 \mu \mathrm{M})$ in aqueous solutions after an infrared laser heating pulse, respectively, with and without PNIPAM-5 $\left(C_{\text {PNIPAM }-5}=2 \times 10^{-4}\right.$ $\mathrm{g} / \mathrm{mL}$ ), where $[\mathrm{ANS}]=118 \mu \mathrm{M}$, power of the heating pulse is $6.0 \mathrm{~mJ}$, and the initial temperature before the heating is $30.4^{\circ} \mathrm{C}$. The inset shows a semilogarithmic plot of $(1-f)$ vs $t$, where $f$ is defined as $\left[I_{\mathrm{F}}(t)-I_{\mathrm{F}}(0)\right] /\left[I_{\mathrm{F}}(\infty)-I_{\mathrm{F}}(0)\right]=1-A \exp \left(-t / \tau_{\text {fast }}\right)-(1-A) \exp -$ $\left(-t / \tau_{\text {slow }}\right)$.

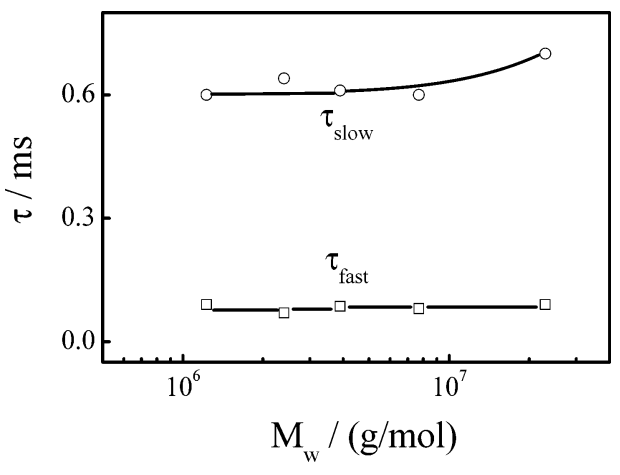

Figure 2. Chain length dependence of two characteristic times related to two transition stages, where PNIPAM concentration was kept at 2 $\times 10^{-4} \mathrm{~g} / \mathrm{mL}$ and $[\mathrm{ANS}]=118 \mu \mathrm{M}$.

stages. To fit such a two-stage transition, we used a doubleexponential function with two characteristic transition times $\left(\tau_{\text {fast }}\right.$ $=0.11 \pm 0.01 \mathrm{~ms}$ and $\tau_{\text {slow }}=0.83 \pm 0.06 \mathrm{~ms}$ ). Each straight line in the inset represents one exponential term. According to Kikuchi et al., ${ }^{23}$ the average $\langle\tau\rangle$ should be in the order of a few milliseconds for the chains used, and the characteristic time of the initial pearl formation would be only a few percent of $\langle\tau\rangle$. Clearly, $\tau_{\text {fast }}$ and $\tau_{\text {slow }}$ are in the reasonable ranges, but shorter than the previous reported values. ${ }^{18}$ Note that in the previous stopped-flow measurements ${ }^{18}$ the coil-to-globule transition was induced by mixing water with methanol since the pyrene-labeled PNIPAM copolymer is not soluble in pure water. The mixing of water and methanol is much slower that the 20-ns heating laser pulse. As discussed before, the initial pearl formation is localized on the chain, and its characteristic time should be independent of the chain length.

Figure 2 shows that $\tau_{\text {fast }}$ is indeed independent of the chain length, consistent with previous predictions of Halperin et al. ${ }^{22}$ and Kuznetsov et al. ${ }^{20}$ Therefore, the study of the chain length independence of $\tau_{\text {fast }}$ is a critical confirmation that the fast process in Figure 1 is related to the initial pearl formation. It is also expected that $\tau_{\text {slow }}$ is a weak function of the chain length since it is scaled to $N$ as $\tau \sim N^{1 / 5}$ proposed by Halperin and Goldbart. ${ }^{22}$ In order to be sure what we observed in Figure 1 is indeed related to the coil-to-globule transition of individual PNIPAM chains, namely, there is no interchain association during the observation time period $(\sim 4 \mathrm{~ms})$, we further measured the Rayleigh scatting intensity from each PNIPAM aqueous solution by blocking the fluorescence light with a filter.

Figure 3 shows that, unlike the fluorescence intensity, there is no significant change in the Rayleigh scattering intensity, i.e.,

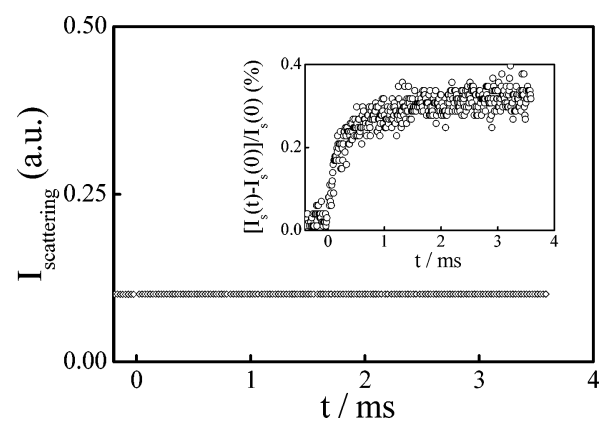

Figure 3. Time dependence of scattering intensity of PNIPAM-5 aqueous solution. The inset shows the ratio of the change of the scattering light intensity $\left[I_{\mathrm{s}}(t)-I_{\mathrm{s}}(0)\right]$ to $I_{\mathrm{s}}(0)$.

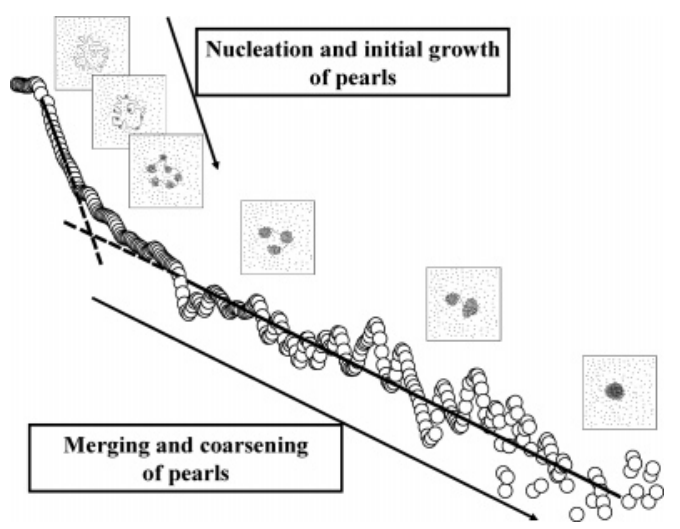

Figure 4. Schematic of two kinetic stages of the coil-to-globule transition of a long linear homopolymer chain in a dilute solution.

no interchain association/aggregation. Otherwise, $I_{\text {scattering }}$ would significally increase because it is proportional to the square of the mass of a scattering object $(M)$; namely, $I_{\text {scattering }} \propto M^{2}$, sensitive to even a small amount of interchain association. A careful examination actually reveals a very small increase of $I_{\text {scattering }}(\sim 0.3 \%)$, as shown in the inset. Such a small increase of $I_{\text {scattering }}$ might reflect that some of ANS molecules were entrapped inside the collapsed chain segments or a small change of the refractive index increment $(\mathrm{d} n / \mathrm{d} c)$ due to the dehydration after the temperature jump. The fitting of such a small increase of $I_{\text {scattering }}$ with a double-exponential equation also results in two distinct transition times: $\tau_{\text {fast }}=0.10 \pm 0.01 \mathrm{~ms}$ and $\tau_{\text {slow }}$ $=0.90 \pm 0.10 \mathrm{~ms}$, consistent with those obtained from the ANS fluorescence intensity measurements.

Our studies (not shown) also revealed that both $\tau_{\text {fast }}$ and $\tau_{\text {slow }}$ are independent of the PNIPAM or ANS concentration. These results further confirm what we observed in Figure 1 is indeed related to the folding of individual PNIPAM chains and that the presence of ANS has no influence on the coil-to-globule transition kinetics in dilute solutions. Further, we varied the intensity of the infrared laser heating pulse to study the quenching depth $(\Delta T)$ effect on the transition kinetics. As expected, the coil-to-globule transition indeed becomes slower when the quenching depth is reduced (the decrease of the jumping temperature).

In summary, using a combination of a fast infrared laser heating pulse-induced temperature jump and the time-dependent fluorescence and Rayleigh scattering intensity measurements, we have, for the first time, revealed that the coil-to-globule transition of individual linear poly $(N$-isopropylacrylamide $)$ homopolymer chains in dilute solutions have two distinct kinetic stages with two characteristic transition times $\left(\tau_{\text {fast }} \sim 0.1 \mathrm{~ms}\right.$ and $\tau_{\text {slow }} \sim 0.8 \mathrm{~ms}$ ). The chain-length-independent first stage 
can be attributed to the nucleation and initial growth of some "pearls" (locally contracting segments) on the chain, while the second and relatively slower process is related to the merging and coarsening of the "pearls". Figure 4 schematically summarizes such a coil-to-globule conformational transition of a homopolymer in a dilute solution after the heating laser pulse. Unlike previous predictions, our experimental results showed no distinction either between the nucleation and initial growth of the pearls or between the packing and merging of the pearls. It should be noted that Figure 4 only reflects what we observed and lacks factual accuracy. To our knowledge, it is not possible to directly see a synthetic polymer chain in the real space at this moment.

Acknowledgment. The financial support of the Hong Kong Special Administration Region Earmarked (Grant No. CUHI4025/ 04P, 2160242), the Chinese Academy of Sciences (Special Grant No. KJCX2-SW-H14), and the Natural National Science Foundation (Project No. 20533070) is gratefully acknowledged. The authors thank Professors Martin Gruebele and Shuqin Yu for their help in the setting up the laser instrument.

\section{References and Notes}

(1) Stockmayer, W. H. Makromol. Chem. 1960, 35, 54

(2) Lifshitz, I. M.; Grosberg, A. Y.; Khokhlov, A. R. Rev. Mod. Phys. 1978, 50, 683 .

(3) de Gennes, P. G. J. Phys., Lett. 1985, 46, L639.

(4) Grosberg, A. Y.; Kuznetsov, D. V. Macromolecules 1992, 25, 1970, 1980, 1991, 1996.

(5) Matsuyama, A.; Tanaka, F. Phys. Rev. Lett. 1990, 65, 341.

(6) Dasmahapatra, A. K.; Kumaraswamy, G.; Nanavati, H. Macromolecules 2006, 39, 9621.

(7) Chu, B.; Park, I. H.; Wang, Q. W.; Wu, C. Macromolecules 1987, 20, 2833. Park, I. H.; Wang, Q. W.; Chu, B. Macromolecules 1987, 20, 1965.

(8) Wu, C.; Zhou, S. Q. Macromolecules 1995, 28, 8381; Phys. Rev. Lett. 1996, 77, 3053.

(9) Winnik, F. M. Macromolecules 1990, 23, 233.

(10) Maeda, Y.; Nakamura, T.; Ikeda, I. Macromolecules 2001, 34, 1391.
(11) Ding, Y. W.; Ye, X. D.; Zhang, G. Z. Macromolecules 2005, 38, 904.

(12) Melnikov, S. M.; Sergeyev, V. G.; Yoshikawa, K. J. Am. Chem. Soc. 1995, 117, 9951.

(13) Yoshikawa, K.; Takahashi, M.; Vasilevskaya, V. V.; Khokhlov, A. R. Phys. Rev. Lett. 1996, 76, 3029.

(14) Sali, A.; Shakhnovich, E.; Karplus, M. Nature (London) 1994, 369, 248.

(15) Chu, B.; Ying, Q. C.; Grosberg, A. Y. Macromolecules 1995, 28, 180. Chu, B.; Ying, Q. C. Macromolecules 1996, 29, 1824.

(16) Nakamura, Y.; Sasaki, N.; Nakata, M. Macromolecules 2001, 34, 5992.

(17) Kayaman, N.; Gürel, E. E.; Baysal, B. M.; Karasz, F. E. Macromolecules 1999, 32, 8399.

(18) Xu, J.; Zhu, Z. Y.; Luo, S. Z.; Wu, C.; Liu, S. Y. Phys. Rev. Lett. 2006, 96, 027802 .

(19) Byrne, A.; Kiernan, P.; Green, D.; Dawson, K. A. J. Chem. Phys. 1995, 102, 573.

(20) Kuznetsov, Y. A.; Timoshenko, E. G.; Dawson, K. A. J. Chem. Phys. 1995, 103, 4807; 1996, 104, 3338.

(21) Klushin, L. I. J. Chem. Phys. 1998, 108, 7917.

(22) Halperin, A.; Goldbart, P. M. Phys. Rev. E 2000, 61, 565.

(23) Kikuchi, N.; Ryder, J. F.; Pooley, C. M.; Yeomans, J. M. Phys. Rev. E 2005, 71, 061804.

(24) Stryer, L. Science 1968, 162, 526.

(25) Turner, D. H.; Flynn, G. W.; Sutin, N.; Beitz, J. V. J. Am. Chem. Soc. 1972, 94, 1554.

(26) Turner, D. H.; Flynn, G. W.; Lundberg, S. K.; Faller, L. D.; Sutin, N. Nature (London) 1972, 239, 215.

(27) Ameen, S. Rev. Sci. Instrum. 1975, 46, 1209

(28) Williams, A. P.; Longfellow, C. E.; Freier, S. M.; Kierzek, R.; Turner, D. H. Biochemistry 1989, 28, 4283.

(29) Kazzaz, A.; Ruschin, S.; Shoshan, I.; Ravnitsky, G. IEEE J. Quantum Electron. 1994, 30, 3017.

(30) Ballew, R. M.; Sabelko, J.; Reiner, C.; Gruebele, M. Rev. Sci. Instrum. 1996, 67, 3694.

(31) Yamamoto, K.; Mizutani, Y.; Kitagawa, T. Biophys. J. 2000, 79 485.

(32) Wang, J. P.; Gan, D. J.; Lyon, L. A.; El-Sayed, M. A. J. Am. Chem. Soc. 2001, 123, 11284 .

(33) Chu, B. Laser Light Scattering, 2nd ed.; Academic Press: New York, 1991.

(34) Shastry, M. C. R.; Udgaonkar, J. B. J. Mol. Biol. 1995, 247, 1013.

(35) Kumar, Y.; Muzammilt, S.; Tayyab, S. J. Biochem. 2005, 138, 335. MA070167D 